\title{
PREPARATION OF COPPER ELECTRICAL ENGINEERING ALLOY STRIP WITH HIGH SERVICE PROPERTIES
}

\author{
P. A. Vasilevskii, ${ }^{1}$ L. M. Zheleznyak, ${ }^{2}$ \\ M. Yu. Borodin, ${ }^{2}$ and K. Yu. Mal'tseva ${ }^{2}$
}

UDC 669.35-442:621.313.047.2:006.354

Output is organized for industrial batches of electrical engineering strip with improved service properties, i.e., copper bus bars of rectangular section $\left(\sigma_{u}\right.$ determination is simplified and surface quality evaluation after bend testing is improved), commutator copper-cadmium strip of trapezoidal section (hardness increased, strip camber reduced, bulges and scratches absent from basic profile), and commutator coppersilver strip (electrical conductivity increased and stable mechanical properties achieved).

Keywords: electrical engineering strip, copper rectangular bus bars, commutator copper-cadmium and copper-silver trapezoidal sections, quality improvement and copper bus bar production labor content reduced, copper-silver alloy chemical composition optimization.

Ways of improving of obtaining good service properties for electrical engineering strip are considered, i.e., copper rectangular medium hard bus bar (simplification of finding $\sigma_{\mathrm{u}}$ and clarification of surface quality evaluation after bend testing as criteria for copper grain size), commutator trapezoidal copper-cadmium strip (increase in hardness, reduction in strip camber, prevention of development of bulges and scratches in sections), and commutator copper-silver strip (increase in electrical conductivity, mechanical property stability). The limited number of articles makes it impossible to reflect to a complete extent production features for manufacturing all forms of semifinished product, in view of which only the main stages of the process and obtaining results are presented.

Soft copper bus bars are used as conductors with high current loads and low working stresses: power supply for electrolyzer baths, distribution panels, control cabinets, etc. With increased mechanical loads, medium hard copper bus bars are used with increased strength properties compared with soft bus bars, but with sufficient ductility. In view of a lack within Russia of standard documentation for medium hard bus bars, they are produced in accordance with the main specifications of the British standard [1]: $\sigma_{\mathrm{u}} \geq 250 \mathrm{MPa} ; \delta=10-15 \%$; absence of cracks with bending at an angle of $180^{\circ}$ around a mandrel with a radius equal to bus bar thickness; the last requirement should indicate moderate copper grain size. However, there are difficulties in performing tests for these specifications.

In order to prepare standard specimens with the aim of determining $\sigma_{\mathrm{u}}$ and $\delta$, it is necessary to select sections of bus bars, cut into billets, and specimen manufacture. For this, an appropriate machine inventory and tool facilities, standards, additional consumption of electrical energy, etc., are required in production areas (for example, within a central plant laboratory). Another disadvantage is the low efficiency of evaluating the surface condition for presence of cracks with bending bus bar at an angle of $180^{\circ}$, i.e., due to the fact that absence of visible cracks with an unaided eye does not guarantee a spec-

\footnotetext{
${ }^{1}$ Kamkor, Kamensk-Uralskii, Russia.

${ }^{2}$ Yeltsin Ural Federal University (UrFU), Ekaterinburg, Russia; e-mail: bsp@mtf.ustu.ru.
}

Translated from Metallurg, No. 4, pp. 103-108, April, 2013. Original article submitted April 20, 2012. 
ified surface quality (and correspondingly the proper metal grain size) due to the presence in the bending area of a coarse, uneven surface finish, worsening the finished form of a conductor after bending bus bars.

Thus, conditions arise for subjective evaluation of test results in bending, which generate uncertainty in certifying objects. With the aim of considerable simplification of determining surface properties of medium hard copper bus bars and also for satisfying user specifications with respect to their ductility by limiting grain size, the authors have proposed controlling:

- bus bar Brinell hardness in the range 625-770 MPa;

- roughness parameters for the outer bending arc of a bus bar $R_{\mathrm{a}} \leq 2.5 \mu \mathrm{m}$.

A simplified method for determining ultimate strength includes the following. It is well known that there is an extremely simple, but very precise, relationship between $\mathrm{HB}$ and $\sigma_{\mathrm{u}}$, and for copper $\sigma_{\mathrm{u}}=0.48 \mathrm{HB}$ [2]. Analysis of numerous plant tests for determining the ratio of $\sigma_{\mathrm{u}}$ and $\mathrm{HB}$ for copper has shown that deviation from coefficient 0.48 is in total $\pm(0.4-0.5) \%$. This makes it possible to simplify considerably $\sigma_{\mathrm{u}}$ determination by measuring $\mathrm{HB}$, since in this case instead of standard rupture specimens untreated sections of copper bus bar are entirely acceptable. Thus, for medium hard bus bars the value of lower HB limit is $250 / 0.48=521 \mathrm{MPa}$. In order to guarantee the stability of strength and ductility properties for industrial batches of medium hard bus bar, the following ranges should be set: $\sigma_{\mathrm{u}}=300-370 \mathrm{MPa}, \mathrm{HB}=625-770 \mathrm{MPa}, \delta=10-15 \%$, and this relates entirely to the standard specifications. The correlation provided above between $\sigma_{\mathrm{u}}$ and $\delta$ on one hand, and HB on the other hand, confirms the dependences of these properties on relative reduction of area $\varepsilon$, presented graphically, for example in [3].

The level of $\mathrm{HB}=625-770 \mathrm{MPa}$ and the absence of coarse rough surface in the bending area of a bus bar is achieved by drawing hot pressed billets in two passes with complete light annealing between them in a conveyor furnace with water seals at $t=650 \pm 30^{\circ} \mathrm{C}$. In the first pass, $\varepsilon=20-28 \%$ is adopted, which is due to significant tolerance for pressed billet thickness $( \pm 0.5 \mathrm{~mm})$ as a result of giving the cross section of the pressing die channel a double-convex shape with the major side of a rectangle with the aim of compensating for a negative effect of channel "floating" caused by action of high contact stresses at the pressing temperature. Designation in the finishing pass of $\varepsilon=7-10 \%$ (this is lower than with preparation of hard copper bus bars) is due, on one hand to a requirement of maintaining the lower limit $\delta=10 \%$, and on the other hand a requirement for achieving the required values of $\sigma_{\mathrm{u}}$, or value of $\mathrm{HB}$ equivalent to it.

It is well known that ductility properties of metal product are closely connected with grain size: an increase in ductility is promoted by grain refinement [4, etc.]. A favorable result of qualitative evaluation of the level of copper bus bar ductility after bend testing at an angle $180^{\circ}$ around a mandrel [1], i.e., absence of cracking in the area of a bend, is complicated to achieve. However, retention after this test of an original bus bar surface condition is almost impossible as a result of cold plastic deformation in the area of a bend, and as noted it acquires a form of lumpy surface given the name "orange peel" [5]. In source [5], it is shown that "this effect consists of forming at the surface waviness, visible to the unaided eye and caused by a coarse metal grain size." This surface condition is identified visually with optical instruments, and for copper shaped sections this surface is sometimes called "erysipelous" [6].

With the aim of avoiding visual evaluation of surface condition as a ductility criterion and transition to a more reliable procedure, the authors have proposed and proven in practice limitation of surface roughness over the outer arc of the area of a bus bar bend with the value $R_{\mathrm{a}} \leq 2.5 \mu \mathrm{m}$. Bus bar roughness is not controlled by the RF standard [7], but it should be superimposed on the double tolerance for cross section size: for bus bar $5 \times 50 \mathrm{~mm}$ this tolerance for $5 \mathrm{~mm}$ thick is $50 \mu \mathrm{m}$. Consequently, limitation of roughness by an upper limit $R_{\mathrm{a}}=2.5 \mu \mathrm{m}$ (according to GOST 2789-88 gradation) is the main and entirely satisfactory specification for customers, and this evaluation is provided according to GOST 2789-88 by means of an instrument for measuring roughness, simple in operation, and requiring minimum time expenditure.

With observation of the cited technological regulation, actual values of roughness over the outer arc in the area of a bus bar bend at an angle of $180^{\circ}$ were 1.8-2.1 $\mu$ m, i.e., guaranteed not to exceed the proper level $R_{\mathrm{a}}=2.5 \mu \mathrm{m}$. Thus, a reduction in labor content and simplification for determining the level of qualitative and quantitative properties of medium hard bus bars are achieved, both for the producer and user.

Commutator strips of trapezoidal cross section (Fig. 1) of electrical engineering copper alloys (in particular, cadmium bronze BrKd1) in electrical engineering plants are subject to cold stamping and segments are prepared that are the main commutator components, i.e., the most important assembly of an electrical machine, on which stable operation reliability depends. The dimensions and section angle for commutator strips, are not standardized, since choice of a required 


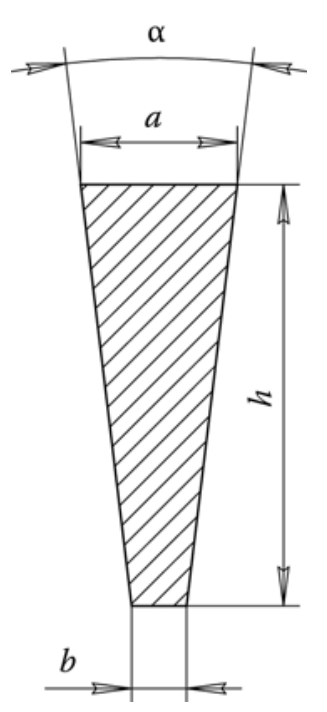

Fig. 1. Commutator strip profile.

shape, providing the necessary electrical parameters, is carried out after appropriate calculation. This situation generates continuous expansion of the range of commutator shapes, and currently thousands of items. In assembling (forming) a commutator, several tens of segments are used, insulated from each other by micanite (mainly mica) interlayers, and therefore it is easy to state that if the angle of a section does not correspond to tolerances, it is impossible to assemble a compact high quality commutator.

Taking account of the action on the commutator of a number of factors of mechanical and electrical nature, this material should exhibit adequate hardness in order to withstand wear; an increased softening temperature, recrystallization threshold, and stress-rupture strength; high creep resistance, necessary for hot forming of the commutator, and also (especially) for machines operating at $180-200^{\circ} \mathrm{C}$.

Realization of the whole set of properties listed is extremely difficult; therefore, as a rule a compromise solution is found, and then the commutator operating life and the electrical machine itself will depend on how alloy answers specific operating conditions. Commutator strips of cadmium bronze BrKd1, according to the standard [8], should satisfy the following specifications: $\mathrm{HB} \geq 950 \mathrm{MPa}$; strip camber $\leq 4 \mathrm{~mm}$ per $1 \mathrm{~m}$ length; magnitude of longitudinal bulges and scratches in a section $\leq 0.4 \mathrm{~mm}$; thin base $\geq 3 \mathrm{~mm}$ with a segment height of $>105 \mathrm{~mm}$; accuracy classes, i.e., normal, improved, high. From analysis of this information, a very high level of specifications follows [8] for segment geometric parameters, accuracy, and hardness. However, strips for electrical machines operating under heavy-duty operating conditions (in particular for traction machines of mainline electric and diesel engines) and even more rigid specifications are laid down [9]: HB $\geq 1000 \mathrm{MPa}$; strip camber $\leq 1.5 \mathrm{~mm}$ per $1 \mathrm{~m}$ length; magnitude of longitudinal bulges and scrathces $\leq 0.2 \mathrm{~mm}$; thin base $\geq 2 \mathrm{~mm}$; high accuracy class.

As a result of industrial test studies and practical use of the production procedure [10], the following high values of commutator segment parameters have been achieved:

1. $\mathrm{HB}=1000-1120 \mathrm{MPa}$ by increasing the reduction factor for thickness in the finishing drawing pass to $\mu=1.27-1.30$ (previously used $\mu=1.25$ ); with $\mu>1.27$, the minimum hardness level (1000 MPa) is not achieved; with $\mu>1.30$, a marked reduction in alloy ductility properties complicates treatment of surplus hard strips for a plant user.

2. The absence of trapezoidal cross section strip camber solely with observance of the equality $\mu_{\mathrm{a}}=\mu_{\mathrm{b}}$ in the finishing drawing pass (where $\mu_{\mathrm{a}}$ and $\mu_{\mathrm{b}}$ are reduction factors for the minor and major bases of a segment (see Fig. 1)). It is very complicated to provide this equality under production conditions due to the broad field of tolerance $( \pm 0.7 \mathrm{~mm})$ for thickness of pressed billets; nonuniform wear of working drawing die channels used as a minimum for three drawing passes; difficulties of treatment and adjustment of the drawing channel with the use of cermet hard alloy drawing dies. 


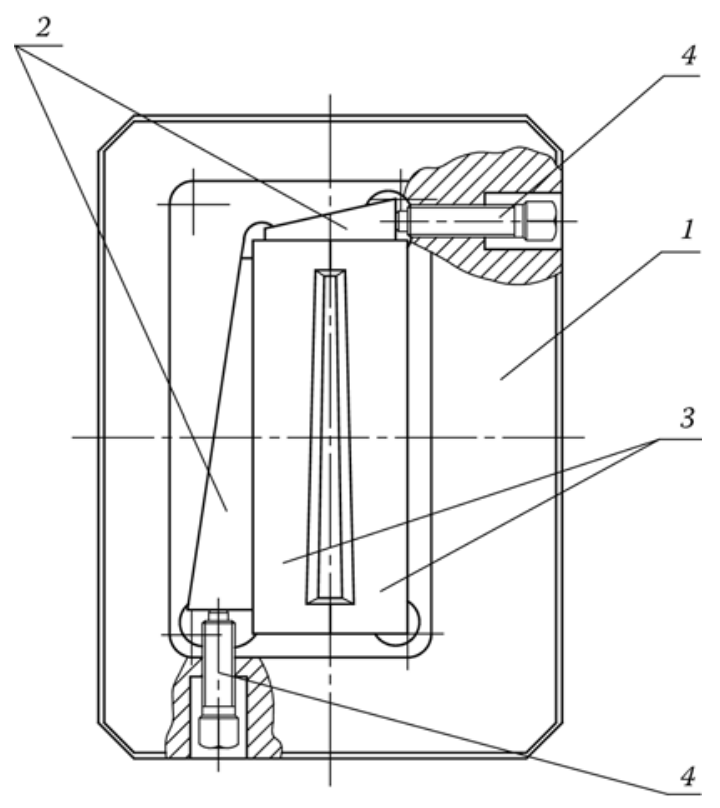

Fig. 2. Composite drawing die for preparing commutator strip: 1) casing;

2) wedges; 3) working inserts; 4) screws.

Apart from this, a ponderable reason for strip camber is the double-convex shape of the pressing die channel cross section described above (in the section concerning copper bus bars). These factors also probably serve as a basis for designating high standard for strip camber, i.e., $4 \mathrm{~mm}$ for $1 \mathrm{~m}$ length. Actually markedly lower values of strip camber for strip in the range $1.0-1.5 \mathrm{~mm}$ per $1 \mathrm{~m}$ length were achieved as follows:

first, from a previous method for making die channels (as a rule, from steel $3 \mathrm{Kh} 2 \mathrm{~V} 8$ by electric-arc treatment followed by manual finishing) changed to an advanced method of electro-erosion cutting in a precision AGIECUT CLASSIC $2 S$ (Switzerland) machine using high precision brass wire as a consumable electrode. This considerably increased die channel dimensional precision;

second, die material used was a highly efficient alloy, nimonic 80 , significantly more heat-resistant compared with steels, and not inclined towards "floating"; and

third, drawing was carried in composite drawing dies with hard alloys inserts (Fig. 2), whose finishing was carried out in a surface grinder, and therefore their dimensions were quite simply corrected. There was an increase in drawing tool life and its adjustment stability.

3. Working C-shaped inserts of a composite drawing die are closely adjacent to each other over the ground surfaces and fixed in a rigid frame by wedges and screws. This provides preparation of a commutator segment without bulges and scratches in its bases. However, with prolonged drawing die operation there is an unfavorable effect of the following factors; insert wear as a result of high pressure, acting normal to the channel surface; harmful factors probable under production conditions (inadequate lubricant supply to a channel or a reduction in its quality; entry of scale and contaminants into the channel, dynamic loads on a tool at the instant of starting a drawing machine, etc.). An insignificant gap may arise in the insert contact planes, and therefore with the aim of excluding in the segment bases even minimal bulges and scratches during insert preparation inclination of planes in contact to the drawing axis is provided.

4. Limitation of the minimum thickness of a thin base to the value of $3 \mathrm{~mm}[8]$ is predicted by obvious production difficulties for section manufacture with a thinner base. Preparation of very thin (up to $1 \mathrm{~mm}$ ) base with a segment height of $h>105 \mathrm{~mm}$ is possible in principle, but for this up to seven to eight drawing passes are required with associated subsidiary operations, i.e., annealing, pickling, flaring of grips, etc. This prolongs the production cycle and causes such an increase in product cost that the results achieved become economically undesirable; this segment may only be manufactured to individ- 
TABLE 1. Chemical Composition of MS0.1 and Copper MOk, wt.\%, Not More Than

\begin{tabular}{|c|c|c|c|}
\hline \multirow{2}{*}{ Element } & \multicolumn{2}{|c|}{ Alloy MS0.1 } & Copper M0k \\
\cline { 2 - 4 } & TU 48-21-137-88 [15] & DIN 17666 [16] & GOST 859-2001 [17] \\
\hline $\mathrm{Ag}$ & $0.08-0.12$ & $0.08-0.12$ & 0.002 \\
\hline $\mathrm{Bi}$ & 0.001 & - & 0.0005 \\
\hline $\mathrm{Sb}$ & 0.002 & - & 0.001 \\
\hline $\mathrm{As}$ & 0.002 & - & 0.001 \\
\hline $\mathrm{Fe}$ & 0.004 & - & 0.001 \\
\hline $\mathrm{Ni}$ & 0.002 & - & 0.001 \\
\hline $\mathrm{Pb}$ & 0.004 & - & 0.002 \\
\hline $\mathrm{Sn}$ & 0.002 & - & 0.002 \\
\hline $\mathrm{S}$ & 0.004 & - & 0.015 \\
\hline $\mathrm{O} 2$ & 0.0035 & - & 0.001 \\
\hline $\mathrm{Zn}$ & 0.004 & - & 0.001 \\
\hline $\mathrm{P}$ & 0.002 & Balance & 99.97 \\
\hline $\mathrm{Cu}$ & Balance & 0.05 & 0.03 \\
\hline Total impurities & 0.05 & & \\
\hline
\end{tabular}

ual order. However, a tendency of contemporary electrical engineering is the fact that realization of high service properties for critical purpose machines of user enterprises, apart from other requirements, prescribe a condition of reducing established standard of minimum thickness of the minor base $(3 \mathrm{~mm})$. Regimes developed and accomplished in practice provide preparation of the base of a segment with a minimum thickness of $2 \mathrm{~mm}$.

Thus, the technical level of commutator strips made of cadmium bronze corresponds to the highest quality category, i.e., the problem is resolved of a marked improvement in user product properties.

Alloying copper with silver even in small amounts markedly improves (compared with electrical engineering copper) object service properties: electrical conductivity, recrystallization threshold, stress-rupture strength, and creep resistance [3, 11]. The advantage of copper-silver alloy over the broadly used cadmium bronze within Russia is the fact that it is ecologically friendly.

The proper hardness level (HB $\geq 1200 \mathrm{MPa}$ ) [12] for commutator strips made of copper-silver alloy MS0.1 is connected with heavy-duty operating conditions of the electrical machines mentioned above. During operation, and during assembly (forming) a commutator is subjected to considerable mechanical loads and repeated heating and cooling cycles [13, 14];

1) during pressing in a special attachment segments and micanite plates it is heated twice to $180-220^{\circ} \mathrm{C}$;

2) after turning a commutator in an assembly it is heated twice to $150-160^{\circ} \mathrm{C}$ and pressed again;

3 ) then it is speeded up twice with a rate 1.25 times greater than $n_{\max }$ with simultaneous heating to $130-150^{\circ} \mathrm{C}$; with the use of an armature with organosilicon insulation $t=180-200^{\circ} \mathrm{C}$; and

4) finally, during soldering in a bath with molten solder protruding parts of a commutator (risers) are strongly heated.

With satisfactory specific electric resistance strips of alloy MS0.1 $\rho=0.01850 \Omega \cdot \mathrm{mm}^{2} / \mathrm{m}$ [12] for heavy-duty user operating conditions (for example Silovye Mashiny, St. Petersburg) additional specifications are laid down for a marked reduction in electrical resistance. Thus, commutator strips of alloy MS0.1 should satisfy two opposite specifications: on one hand, exhibit sufficiently high $\sigma_{\mathrm{u}}$ and $\mathrm{HB}$, including at temperatures up to $300^{\circ} \mathrm{C}$, providing apart from other factors a specific level of impurity content, and, on the other hand, high electrical conductivity, achieved by a reduction in their content. 
In particular, strips at room temperature should have $\sigma_{\mathrm{u}} \geq 294 \mathrm{MPa}, \mathrm{HB} \geq 950 \mathrm{MPa}, \delta \geq 5 \%$ [12]; after heating to $300^{\circ} \mathrm{C}$, soaking for $1 \mathrm{~h}$ and cooling to the ambient temperature $\sigma_{\mathrm{u}}, \mathrm{HB}$, and $\delta$ should be not less than $274 \mathrm{MPa}, 900 \mathrm{MPa}$, and $7 \%$, respectively; electrical conductivity should remain at the level indicated above.

From analysis of data provided in Table 1, very strict specifications follow for chemical composition of copper M0k [15] and alloy MS0.1, laid down by the Russian TU [12, 16], and also a German standard [17]. With the aim of improving electrical conductivity and stable mechanical properties of objects, a study has been carried out (with simultaneous output of industrial test batches) for varying impurity element content (with observation of their overall sum of not more than $0.05 \%$ ). As a result of this, the following have been achieved: tolerance of their favorable effect on alloy properties and approach of its composition to the optimum, i.e., from the position of successful combination of mechanical and electrical properties. Analysis of the effect of impurities on alloy properties and on features of object manufacturing technology based on laboratory studies, and also according to information in [3,11], has shown that the effect of $\mathrm{Ni}, \mathrm{Al}$, and $\mathrm{Mn}$ impurities is most positive for strength and ductility properties of copper-silver alloy (including at up to $300^{\circ} \mathrm{C}$ ). Since $\mathrm{Al}$ and $\mathrm{Mn}$ are not present in practically significant amounts in cathode copper M0k (as a basis of alloy MS0.1), then the main element improving object operating properties to a significant extent should be acknowledged as nickel. In view of this, its content in the alloy, compared with cathode copper, was increased by a factor of two (see Table 1). An increase in iron impurity content, as practice has shown, does not reduce electrical conductivity, but combined with other elements it increases semifinished product strength properties. A reduction in content of harmful oxygen impurity (fourfold) and limitation of phosphorus content was achieved as follows: instead of the widely accepted deoxidation of melt with copper phosphide it was carried out with active stirring of a melt in a small capacity furnace, and also with the use of calcined wood charcoal as a cover. Concerning limitation of the content of such harmful (from the point of view of worsening ductility properties) impurities as $\mathrm{Bi}, \mathrm{Pb}$, and $\mathrm{S}$ [3], then this area of optimizing alloy properties is also predicated by considerations aimed at resolving this problem.

The properties of commutator strips made of copper-silver alloy MS0.1, in particular, the two highest tonnage items $2.05 \times 3.70 \times 92 \mathrm{~mm}$ and $2.11 \times 4.34 \times 102 \mathrm{~mm}$, manufactured in accordance with the procedure developed, fully correspond to the standard TU [12], in fact: $\sigma_{\mathrm{u}}=327-334 \mathrm{MPa}, \delta_{5}=8.3 \%, \delta_{10}=4.5 \%, \mathrm{HB}=955-980 \mathrm{MPa}$, and $\rho=0.01742 \Omega \cdot \mathrm{mm}^{2} / \mathrm{m}$.

Conclusion. Alloy of corrected chemical composition approximates the optimum, and fully meets the specifications of standard documents for the level of operating properties and simultaneously makes it possible to reduce manufacturing delays in a producer enterprise.

\section{REFERENCES}

1. BS1432:1977, Specification for Copper for Electrical Proposes, Rod and Bar, British Standard Institution, London.

2. P. P. Kostin, Physicomechanical Testing of Metals, Alloys, and Nonmetallic Materials [in Russian], Mashinostroenie, Moscow (1990).

3. A. P. Smiryagin, N. A. Smiryagin, and V. A. Belova, Commercial Nonferrous Metals and Alloys [in Russian], Metallurgiya, Moscow (1974).

4. V. S. Zolotarevskii, Mechanical Properties of Metals [in Russian], MISiS, Moscow (1998).

5. $\quad$ Orange Peel Effect, www.juvelinetec/process/process.htm?id=mec obr 2.

6. $\quad$ TU 16-501.011-73, Shaped Electrical Engineering Section, Group V55, Code OKP 184480.

7. GOST 434-78, Copper Wire of Rectangular Section and Copper Bus Bars for Electrical Engineering Purposes, Izd. Standartov, Moscow (1989).

8. GOST 4134-75, Copper Alloy Sections for Electrical Machine Commutators, Izd. Standartov, Moscow (1980).

9. TU 48-21-491-75, Commutator Strips Made of Copper with Cadmium, Reg. No. 138862 of 07.21.75, Group V53, Code OKP 184480.

10. N. S. Arsent'eva, N. F. Bokov, O. N. Dashkevich, and L. M. Zheleznyak, RF Patent 65892 for useful model, IPC C220C20/00, Commutator Strip of Cadmium Bronze, subm. 07.11.2005, publ. 08.27.2007, Byull., No. 24.

11. A. A. Presnyakov and V. V. Chervyakova, Complex Bronzes and Brasses [in Russian], Nauka, Alma-Ata (1974). 
12. TU 16-501.033-87, Sections of Trapezoidal Shape for Electrical Engineering Machine Commutator Plates, Group V52, Code OKP 184480.

13. A. B. Ioffe, Traction Electrical Machines [in Russian], Gosenergoizdat, Moscow-Leningrad (1957).

14. N. M. Batalov, V. A. Belyi, A. B. Ioffe, et al., Metallurgical Crane Electric Motors [in Russian], 2nd. ed., Energiya, Moscow (1967).

15. GOST 859-2001, Copper. Grade M, Izd. Standartov, Group V51.

16. TU 48-21-137-76, Copper Ingots, Alloyed with Silver, Group V51, Code OKP 1733220500 (1988).

17. DIN 17666, Niedriglegierte Kupfer-Knetlegierungen, DK 669.35.018.26-4, December (1983). 